\title{
Targeted deep sequencing contributes to guiding personalized targeted therapy for advanced biliary tract cancer patients with non-radical resection: A real-world study
}

\author{
FEILING FENG $^{1 *}$, QINGBAO CHENG $^{1 *}$, DADONG ZHANG ${ }^{2 *}$, BIN LI $^{1}$, HAO QIN ${ }^{2}$, CHANG XU $^{1}$, \\ MIAO HAN ${ }^{2}$, YONG YU ${ }^{1}$, ZHIZHEN LI ${ }^{1}$, JING-YU LI ${ }^{2}$, ZHIQUAN QIU ${ }^{1}$, LEI XIONG ${ }^{2}$, \\ CHEN LIU $^{1}$, FUGEN LI ${ }^{2}$, BIN YI ${ }^{1}$ and XIAOQING JIANG ${ }^{1}$ \\ ${ }^{1}$ Department of Biliary I, Shanghai Eastern Hepatobiliary Surgery Hospital, Navy Military Medical University, \\ Shanghai 200438; ${ }^{2}$ Research and Development Institute of Precision Medicine, 3D Medicines Inc., \\ Shanghai 201114, P.R. China
}

Received June 18, 2019; Accepted January 10, 2020

DOI: $10.3892 /$ or.2020.7491

\begin{abstract}
Targeted therapy based on specific genetic alterations has been proven to be an effective treatment for various types of cancer. In the present study, we aimed to explore the efficacy of personalized targeted therapy guided by targeted deep sequencing for patients with advanced biliary tract cancer (BTC) after non-radical resection. Targeted deep sequencing was performed on 49 patients with BTC, to whom biologic agents were recommended. Among 32 patients with stage IV and R2 resection (a non-radical resection), 21 patients underwent conventional chemotherapy (mGEMOX), while the remaining 11 patients received a personalized targeted agent. The genomic landscape of the 49 patients with BTC was determined and the results showed that genetic alterations were enriched in the ERBB family and cell cycle pathway. After a median follow-up of 12 months, the 11 BTC patients with personalized targeted therapy showed a median progression-free survival (PFS) of 4.5 months (2.5-20.5 months), a median overall survival (OS) of 12.9 months (4.7-24.8 months)
\end{abstract}

Correspondence to: Professor Xiaoqing Jiang or Dr Bin Yi, Department of Biliary I, Shanghai Eastern Hepatobiliary Surgery Hospital, Navy Military Medical University, 225 Changhai Road, Shanghai 200438, P.R. China

E-mail: xqjiang_dandaoyike@163.com

E-mail: billyyi11@163.com

*Contributed equally

Abbreviations: BTC, biliary tract cancer; NGS, next generation sequencing; PFS, progression-free survival; OS, overall survival; DCR, disease control rate; GBC, gallbladder carcinoma; TKIs, tyrosine kinase inhibitors; NSCLC, non-small cell lung cancer; $\mathrm{PR}$, partial response; $\mathrm{SD}$, stable disease; $\mathrm{PD}$, progressive disease

Key words: personalized targeted therapy, biliary tract cancer, non-radical resection, targeted deep sequencing, prognosis and a disease control rate (DCR) of $63.6 \%$. In the other 21 BTC patients, who were undergoing conventional chemotherapy, the BTC patients had a median PFS of 1.5 months (0.5-11.6 months), a median OS of 4.1 months (1.3-18.4 months), and a DCR of $33.3 \%$. In addition, $36.4 \%$ of the patients in the personalized targeted therapy group experienced grade $>2$ treatment-related toxicity vs. $19.0 \%$ of patients in the conventional chemotherapy group. This real-world study suggests that targeted deep sequencing contributes to the guidance of personalized targeted therapy based on individual actionable mutations, which may benefit advanced BTC patients undergoing non-radical resection.

\section{Introduction}

Biliary tract cancer (BTC) is a devastating disease of the digestive system with poor prognosis, and mainly includes bile duct carcinoma and gallbladder carcinoma (GBC). The 5-year survival rate is less than $10 \%$ in patients with advanced or metastatic BTCs according to the National Cancer Database of the American College of Surgeons and the Surveillance, Epidemiology, and End Results (SEER) Program (1). The potentially best treatment for patients with BTCs is radical resection, but many patients are not suitable for curative surgery. For patients with advanced BTC undergoing non-radical resection, the prognosis is very poor. Therefore, the exploration of a new strategy of more precise and effective treatment is critical to improve the prognosis of patients with advanced BTCs undergoing non-radical resection.

Precision medicine means matching the right patients with the right drugs. The use of genetic mutation testing is often required prior to issuing a matching prescription. The availability of appropriate molecular profiling is the key to precision medicine in routine clinical settings (2). A few studies have reported genetic mutation profiling in biliary tract tumors (3-7). In intrahepatic bile duct carcinoma, genes from the mitogen-activated protein kinase (MAPK) signaling pathway (8) are frequently mutated, and gene fusions from the fibroblast growth factor receptor (FGFR) family are also 
common (9). The erythroblastic leukemia viral (v-erb-b) oncogene homolog (ERBB) family including epidermal growth factor receptor (EGFR, also known as ERBB1), ERBB2 (also known as HER2), ERBB3 and ERBB4 has a central role in the tumorigenesis and development (10). The ERBB family receptors are able to activate several downstream pathways, including the RAS-ERK and PI3K-AKT pathways (11). In the Chinese population, alterations in the ERBB family and its downstream signaling pathway account for up to $36.8 \%$ of the alterations detected in gallbladder cancer, and further multivariate analyses have revealed that cases with ERBB pathway mutations have worse outcomes (12). Studies in BTC cell lines have confirmed that the ERBB pathway may be a suitable candidate for disruption as part of treatment for BTC patients (13). Many mutated genes in biliary tract tumors, including ERBB2, PIK3CA, FGFR and IDH1, are targets for biological drugs. There are several studies indicating the potential of comprehensive genomic profiling for improving outcomes in advanced BTC patients $(14,15)$. However, the clinical efficacy of personalized targeted therapy based on the genetic alterations found in BTC patients undergoing non-radical resection has not yet been reported. We aim to investigate the efficacy and safety of personalized targeted therapy with excellent potential for actual use based on specific genetic alterations for patients with advanced BTC after non-radical resection in this real-world study.

\section{Materials and methods}

Patients and data collection. The present study was approved by the Ethics Committee of the Shanghai Eastern Hepatobiliary Surgery Hospital, Navy Military Medical University, Shanghai, China (no. EHBHKY2015-02-010). Forty-nine patients with BTC, which was confirmed by surgical pathology, were enrolled for targeted deep sequencing in the Department of Biliary I, Shanghai Eastern Hepatobiliary Navy Hospital from August 2014 to June 2016 (Table I). Exclusion criteria were stage I disease (AJCC 7th edition) (http://aboutcancer. com/AJCC_stage.htm) at diagnosis, no tumor tissue sample available for targeted deep sequencing, disagreement on conducting target deep sequencing, known or current evidence of HIV, pregnant or lactating females. Thirty-two patients had stage IV disease and $\mathrm{R} 2$ resection (Table II). To investigate the impact of targeted medicine on patients with advanced BTC, eligibility criteria included R2 resection of biliary cancer at TNM stage IV, at least one measurable lesion at baseline and the detection of targeted deep sequencing. These BTC patients began drug treatment one month after the operation. For these patients, a regimen of mGEMOX (16) (gemcitabine $900 \mathrm{mg} / \mathrm{m}^{2}$ and oxaliplatin $80 \mathrm{mg} / \mathrm{m}^{2}$ i.v. infusion on days 1 and 8 every 3 weeks until disease progression or intolerable toxicity) was initially proposed by the local multidisciplinary team as the standard first-line chemotherapy (chemotherapy group). In patients found to have at least one targetable variant and who declined chemotherapy, a biological agent was recommended as an alternative treatment. The specific agent was selected according to the potentially targetable altered gene (targeted therapy group). The information of targetable altered genes corresponding to the biological agent is shown (Table SI). When a patient had some targetable altered genes, we recommended the drugs for patients according to different levels of evidence and gave them all the information they required. The highest level of evidence is that targeted drugs addressing specific gene mutations have been approved by the US Food and Drug Administration (FDA) or China Food and Drug Administration (CFDA) in this tumor. The second kind of evidence is that targeted drugs addressing specific gene mutations have been approved by the FDA or CFDA in other tumors. The third kind of evidence is that also drugs specific to this gene mutation are currently being assessed in clinical trials. The weakest evidence is some preclinical data about the relationship of the drug-gene mutation associations. In this real-world clinical study, the patients made decisions themselves according to the availability of specific drugs and what they were able to afford. The recorded data also included clinicopathological features, operative morbidity, drug administration, number of treatment cycles, and therapy-related toxicity (Tables III and IV).

Study assessment. Progression-free survival (PFS), overall survival (OS) and disease control rate (DCR) were used to assess the efficacy of treatments. The PFS of patients was measured as the duration from the beginning of chemotherapy or precision therapy to the time of disease progression [according to RECIST 1.1 (17)] or death. OS was defined as the period from the start of chemotherapy or targeted therapy to death. The PFS and OS were calculated for all enrolled patients (Table SII). Disease control was defined at the first response assessment as indicated by imaging. Response and disease progression were evaluated using the international criteria proposed by the revised Response Evaluation Criteria in Solid Tumors (RECIST 1.1) Committee (17). The grade of treatment-related toxicities was evaluated according to the National Cancer Institute Common Terminology Criteria for Adverse Events (version 3.0) (18), in order to assess the safety of treatments.

Targeted deep sequencing and variant calling. According to our previous study (19), this panel of targeted deep sequencing consisted of 4,557 exons of 365 tumor-associated genes, and 45 introns from 25 genes where frequent gene fusions could be captured in cancer (Table SIII). Targeted deep sequencing was carried out on hybridization-captured, adaptor ligationbased libraries using DNA extracted from 4 formalin-fixed paraffin-embedded (FFPE) sections cut at $10 \mu \mathrm{m}$ from 49 BTC patients. All of the sequencing assays were performed at the 3D Med Clinical Laboratory Co., Ltd. (Shanghai) and successfully passed the Proficiency Testing (PT) on NGS solid tumor (NGSST) developed by the College of American Pathologists (CAP) (https://www.cap.org/). DNA was isolated from FFPE slides containing at least $20 \%$ tumor cells and the library was prepared using IDT Xgen hybridization buffer (Integrated DNA Technologies, Inc.) for capture and sequenced on an Illumina Nextseq 500 (Biostar Technology Ltd.).

Short reads were mapped by BWA 0.7.12-r1039 with default settings (20). Somatic single-nucleotide variants (SNVs) were identified using Mutect 1.1.4 with default settings, and Indels (smaller than $50 \mathrm{bp}$ ) were identified by Pindel v0.2.5b8 and VarScan v2.4.0 with default settings (21-23). For somatic copy number alterations (CNAs), coverage in the tumor was 
normalized to that in the matched control (blood DNA). Our in-house program combined with modified algorithm was applied to call somatic CNAs (24). Actionable genomic alterations were defined as being linked to commercially available targeted therapies or to targeted therapies currently in ongoing clinical trials. To further annotate the genetic variants found in each patient, an in-house manually reviewed clinical database (3D Medicines Inc.) was used to develop plans for targeted therapy.

Statistical methods. The difference in age between the two groups was tested by using the Student's t test. Fisher's exact test was used to assess the significance of differences between groups, such as sex, cancer type, TNM stage, and operative complication. The heatmap was plotted by $\mathrm{R}$ package GenVisR 1.0.2 (25), which was used to demonstrate somatic mutations spectrum across BTC cohorts. Statistical analyses were performed using $\mathrm{R}$ packages. The log-rank test and Kaplan-Meier analyses were performed for PFS and OS. $\mathrm{P}<0.05$ was considered statistically significant. Kaplan-Meier analyses were performed using SPSS statistical software (version 19.0; SPSS Inc.).

\section{Results}

Clinical characteristics of the patients with biliary tract cancer (BTC). In the present study, we enrolled 49 BTC patients with a median age of 59 years (range, 26-72) at diagnosis (Table I) from August 2014 to June 2016 for targeted deep sequencing. These patients consisted of 32 males and 17 females, and were composed of 21 patients with gallbladder carcinoma and 28 with bile duct cancer (21 intrahepatic, 5 perihilar, and 2 distal cholangiocarcinomas). Most patients $(\mathrm{n}=42,85.7 \%)$ had cTNM stage IV disease (AJCC 7th edition) at diagnosis. Out of the 49 patients, 8 patients with BTCs received radical resection $(\mathrm{R} 0)$, while the others $(n=41,83.7 \%)$ received R1/R2 resection or biopsy. Follow-up was completed for all 49 patients.

Genetic alterations are enriched in the ERBB family and cell cycle pathway in patients with BTC. The genomic landscape of the 49 patients established that TP53 $(\mathrm{n}=31,63.3 \%)$ variants were most prevalent, followed by variants in $\operatorname{KRAS}(\mathrm{n}=12$, 24.5\%), ARIDIA (n=6, 12.2\%), PIK3CA ( $\mathrm{n}=6,12.2 \%)$, SMAD4 $(\mathrm{n}=6,12.2 \%), C D K N 2 A(\mathrm{n}=5,10.2 \%)$, and ERBB4 $(\mathrm{n}=5$, $10.2 \%$ ) (Fig. 1). Further analysis of copy number alterations (CNAs) showed low levels of recurrent amplified genes, such as PIK3CA, SMAD4, FGFR3, SRC, PIK3R2, CDK4, ERBB2, and $C D K 6$. Among these genes, PIK3CA, ERBB2, CDK4, and $C D K 6$ may be suitable drug targets for these BTC patients. In 21 patients with gallbladder cancer (GBC), 8 had mutations in the ERBB pathway. Further analysis of all of the alterations demonstrated that these altered genes were highly enriched in the ERBB family or the cell cycle pathway (Fig. 2A and B).

Personalized targeted therapy in advanced BTC patients with $R 2$ resection. Because tumor staging and resection margins can significantly impact the prognosis of patients with BTCs $(26,27)$, only 32 patients who met the eligibility criteria of having stage IV disease and $\mathrm{R} 2$ resection were enrolled in the
Table I. Characteristics of the 49 patients with biliary tract cancer that received surgery.

\begin{tabular}{|c|c|}
\hline Characteristics & Data values \\
\hline Age, median (range) in years & $59(26-72)$ \\
\hline \multicolumn{2}{|l|}{ Sex, $\mathrm{n}$} \\
\hline Male & 32 \\
\hline Female & 17 \\
\hline \multicolumn{2}{|l|}{ Cancer type, $\mathrm{n}$} \\
\hline Gallbladder carcinoma & 21 \\
\hline Cholangiocarcinoma & 28 \\
\hline \multicolumn{2}{|l|}{ AJCC stage, $\mathrm{n}$} \\
\hline I & 0 \\
\hline II & 2 \\
\hline III & 5 \\
\hline IV & 42 \\
\hline \multicolumn{2}{|l|}{ Type of surgical operation, $n$} \\
\hline R0 & 8 \\
\hline $\mathrm{R} 1$ & 8 \\
\hline $\mathrm{R} 2$ & 32 \\
\hline Biopsy & 1 \\
\hline \multicolumn{2}{|l|}{ Treatment, $\mathrm{n}$} \\
\hline Targeted therapy & 14 \\
\hline Chemotherapy & 35 \\
\hline \multicolumn{2}{|l|}{ Follow-up, n } \\
\hline PFS & 49 \\
\hline OS & 49 \\
\hline NGS detection, $\mathrm{n}$ & 49 \\
\hline
\end{tabular}

AJCC, American Joint Committee on Cancer; PFS, progression-free survival; OS, overall survival; NGS, next generation sequencing.

treatment study (Table II). The patient characteristics, age, sex, cancer type, pTNM stage, and operative morbidity, were similar between the targeted therapy and conventional chemotherapy groups (Table II). In the targeted therapy group, 11 patients received corresponding targeted therapy as an alternative to chemotherapy (Tables III and IV). The mutated genes, recommended drugs, drug administration (drug usage, dosage, and cycle), operative morbidity, prognosis, and treatment-related toxicities of the two groups are described in Tables III and IV. The targeted drugs lapatinib, everolimus, dasatinib, imatinib, pazopanib, and regorafenib were administered to 11 BTC patients in the targeted therapy group, while mGEMOX was given to 21 patients in the chemotherapy group. The administration of these targeted agents and chemotherapeutic regimens was performed by the local multidisciplinary team in this study.

According to the revised RECIST guideline (version 1.1), the targeted therapy group had partial response (PR) in 3 patients, stable disease (SD) in 4 patients, and progressive disease (PD) in the remaining 4 patients, while the chemotherapy group had 2 patients with PR, 5 with SD, and 14 with PD (Tables III and IV). The targeted therapy group had a $63.6 \%$ disease control rate (DCR), while the chemotherapy group had a $33.3 \%$ DCR. Moreover, the targeted therapy group had a median PFS of 
Table II. Characteristics of the 32 patients with stage IV biliary tract cancer after R 2 resection.

Characteristics

Targeted

Conventional chemotherapy $(n=21)$

Age, median (range) in years

therapy $(n=11)$

\begin{tabular}{llll}
\hline All patients $(n=21)$ & P-value & With recommendation $(n=13)$ & P-value
\end{tabular}

Sex

Male

$60(26-65)$

$58(35-66)$

0.645

$61(35-66)$

0.897

Female

7

13

1.000

9

1.000

Cancer type

Gallbladder carcinoma

4

8

4

Cholangiocarcinoma

6

10

1.000

5

8

pTNM stage (AJCC 7th edition)

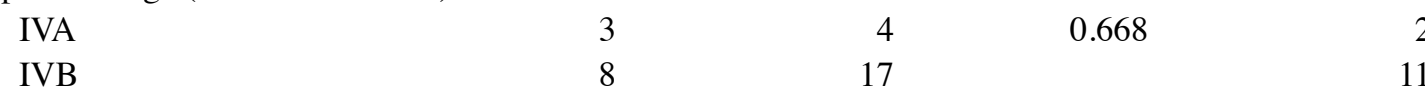

11

0.682

Operative complication

(Clavien-Dindo)

Grade II

10

20

1.000

13

0.458

Grade IIIa

$1+1$

0

AJCC, American Joint Committee on Cancer.

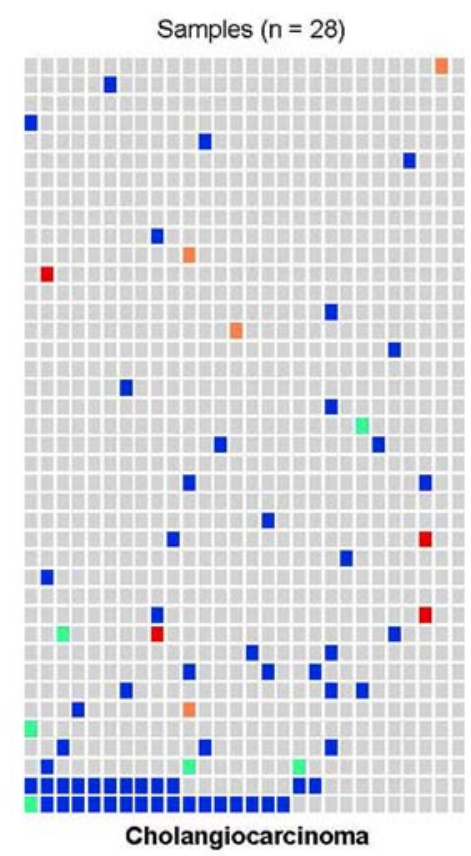

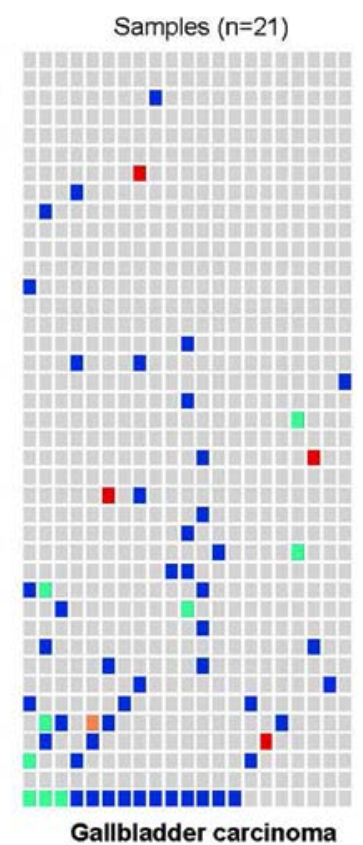

Gallbladder carcinoma

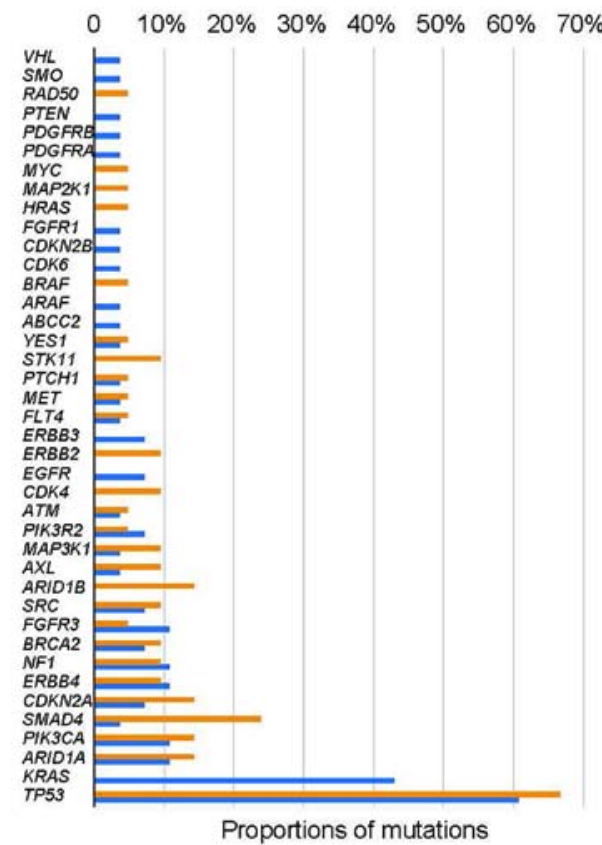

\#ingle nucleotide mutations
Windels Copy loss
WCopy gain

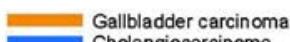

Gallbladder carcinom

Figure 1. Mutational landscape of biliary tract cancers (BTCs). Mutational spectrum of the BTC patients as determined by targeted deep sequencing (left and middle panels). Overall, 28 cholangiocarcinomas and 21 gallbladder cancers were included. The genetic variants landscape showed that TP53, KRAS, ARID1A, and $P I K 3 C A$ were frequently mutated. Mutation subtypes (single nucleotide variant, indel, copy gain and loss) are denoted by color. The right panel shows the frequency of recurrent mutated genes. The histogram with different colors shows the frequency of corresponding genes in cholangiocarcinoma or gallbladder carcinoma, respectively. The colors indicating the frequency of corresponding genes in cholangiocarcinoma and gallbladder carcinoma are reversed in the right panel. $T P 53$, tumor protein P53; KRAS, KRAS proto-oncogene, GTPase; ARIDIA, AT-rich interaction domain 1A; PIK3CA, phosphatidylinositol-4,5-bisphosphate 3-kinase catalytic subunit $\alpha$.

4.5 months (2.5-20.5 months), and a median OS of 12.9 months (4.7-24.8 months) (Fig. 3A and B), while the chemotherapy group had a median PFS of 1.5 months (0.5-11.6 months) and a median OS of 4.1 months (1.3-18.4 months) (Fig. 3C and D). Subgroup analysis of 13 patients from the chemotherapy group who received recommendations of targeted therapy, showed 


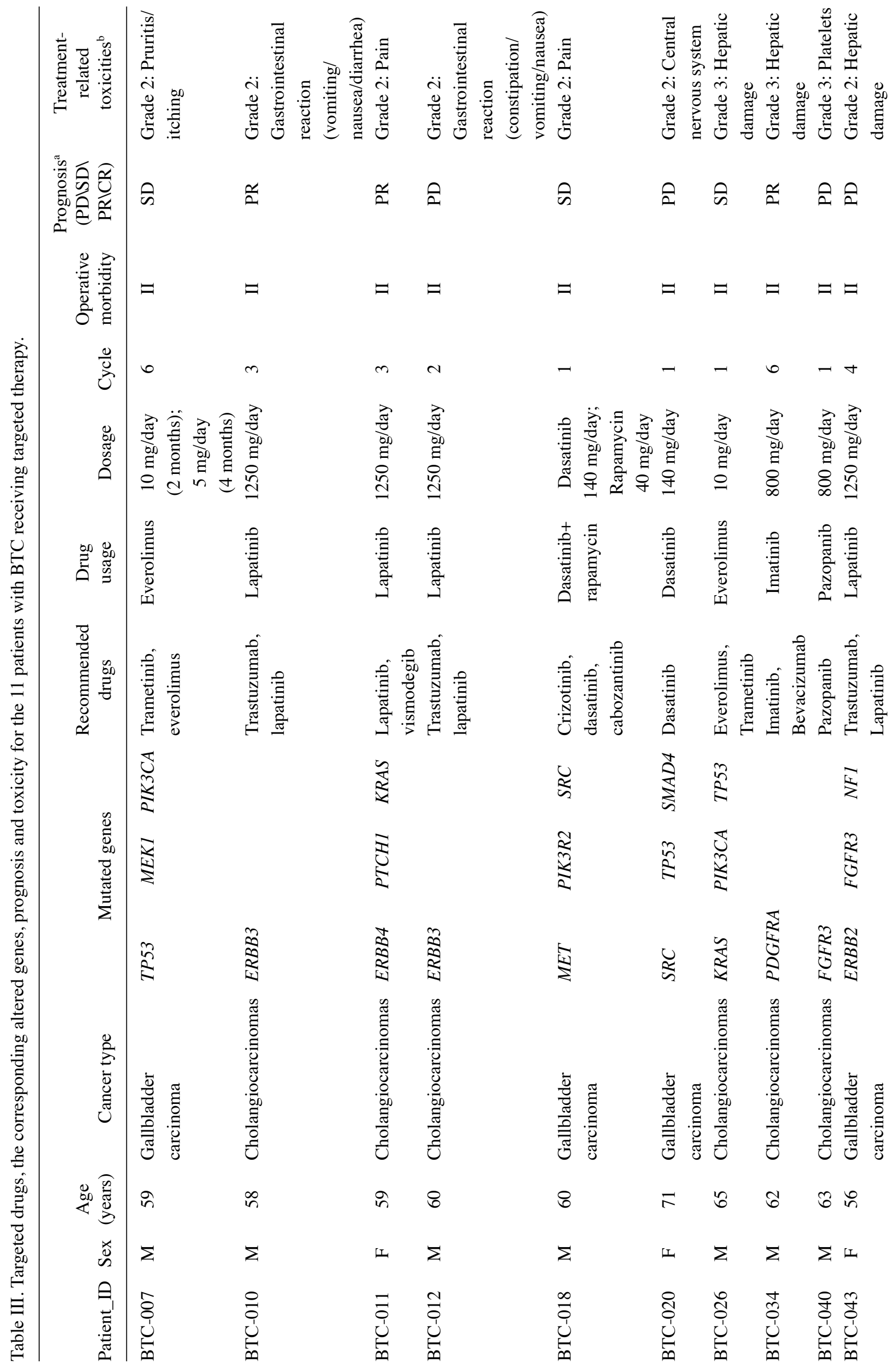


that they had a median PFS of 1.5 months (2.5-20.5 months), a median OS of 2.8 months (4.7-24.8 months) and a DCR of $30.8 \%$ (Fig. S1 and Table IV).

Safety of personalized targeted therapy. In the targeted therapy group, there were 4 patients (BTC-026, BTC-034, BTC-040, and BTC-048) who experienced Grade 3 treatment-related toxicity, including hepatic damage, thrombocytopenia, and fatigue (Table III). The chemotherapy group had 3 patients (BTC-009, BTC-039, and BTC-041) with Grade 3 treatment-related toxicities and 1 patient (BTC-044) with Grade 4 renal impairment (Table IV). In addition, $36.4 \%$ patients in targeted therapy group experienced Grade $>2$ treatment-related toxicity, while $19.0 \%$ patients in the conventional chemotherapy group did.

Three BTC cases of personalized targeted therapy. In this cohort, 11 patients received the corresponding targeted therapies based on their genetic variants. Positive clinical responses were observed in 3 patients and are described herein. A 26-year-old female patient (BTC-048) was diagnosed with Stage IVb Grade 2 gallbladder adenocarcinoma with metastases to the peritoneum in December 2014 (Table III and Fig. S2A). Based on her genetic alterations (Fig. 4A), a targeted drug, regorafenib, targeting RET (160 mg/day) was recommended and was administered on January 25, 2015. On April 21, 2015, the sum of the diameters of all of the measurable lesions was decreased by $29.2 \%$ (SD) according to RECIST 1.1 (Fig. S2B). The patient's performance status was found to have improved a month later, with a reported increase in body weight and decrease in abdominal pain. The patient continued to experience stable disease based on CT imaging (Fig. S2C). However, the patient ceased regorafenib treatment on July 18, 2017 due to Grade 3 fatigue and Grade 2 nausea, vomiting, diarrhea, and stomatitis. On July 30 , the CT images showed PD and the sum of the diameters had increased to the pre-treatment RECIST measurements (Fig. S2D). For this reason, a PFS period of 6 months was recorded for this patient from initiation of treatment in January to disease progression in July (Fig. 4B).

There were also 2 patients with BTC, who received personalized precise therapy with satisfying results. One patient (BTC-010) with stage IVa intrahepatic cholangiocarcinoma who underwent R2 resection, harbored an ERBB3 p.R1127H mutation and the ERBB inhibitor lapatinib was administered (Table III). The PFS and OS of this patient were 20.5 and 24.8 months, respectively. The other intrahepatic cholangiocarcinoma patient (BTC-034), also with stage IVa disease, carried a PDGFRA p.T1066I mutation and was treated with imatinib as recommended (Table III). The PFS and OS were 7.5 and 15.8 months respectively.

\section{Discussion}

In the present study, the landscape of genomic variants in 49 Chinese biliary tract cancer (BTC) patients was obtained. Several studies on whole-exome sequencing and whole genome sequencing of BTCs have recently been published $(3,12,28-30)$. TP53 and KRAS were reported as the mostly frequently mutated genes in previous studies $(9,31)$, and the majority of the variants are single nucleotide variants. These findings 


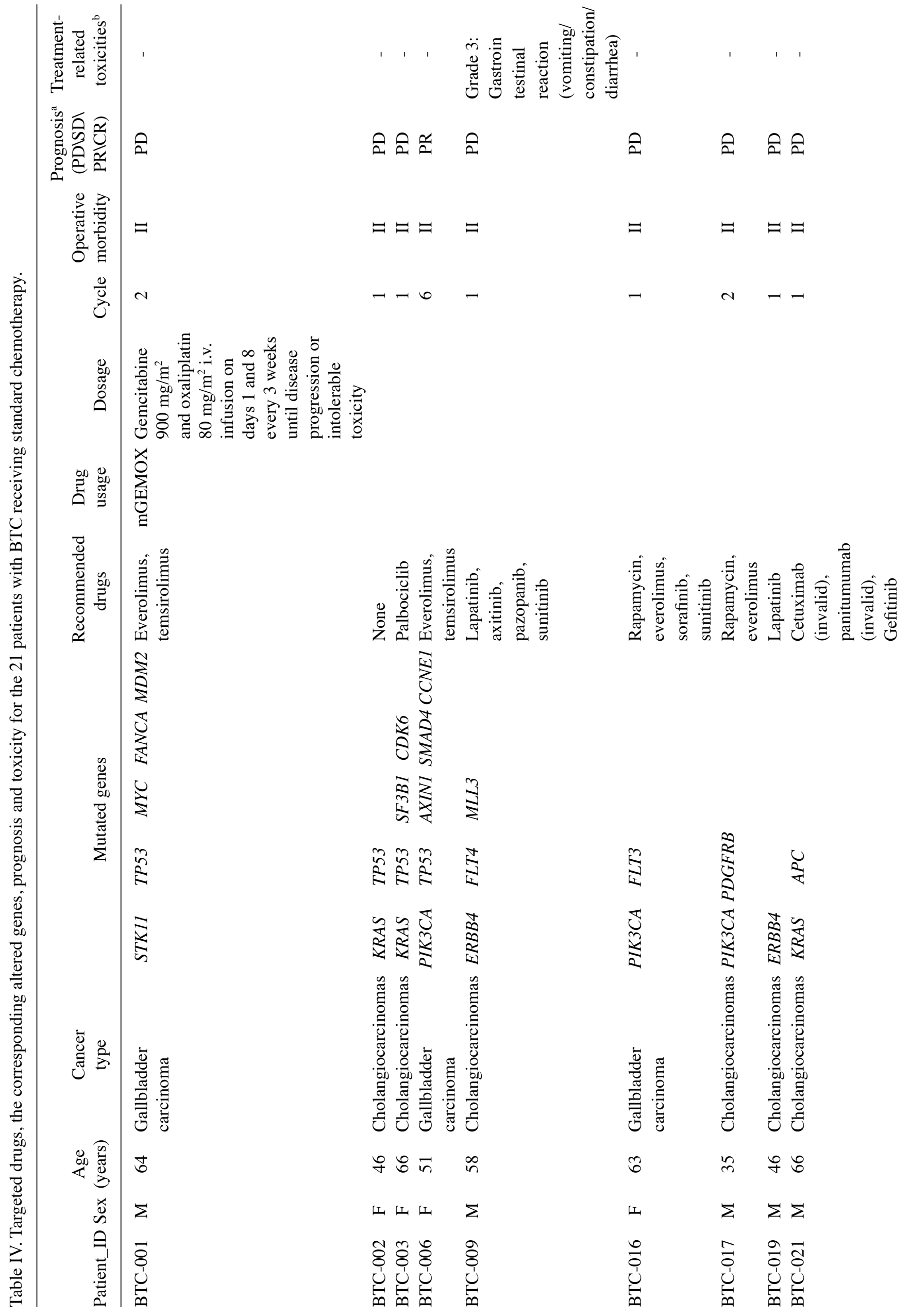




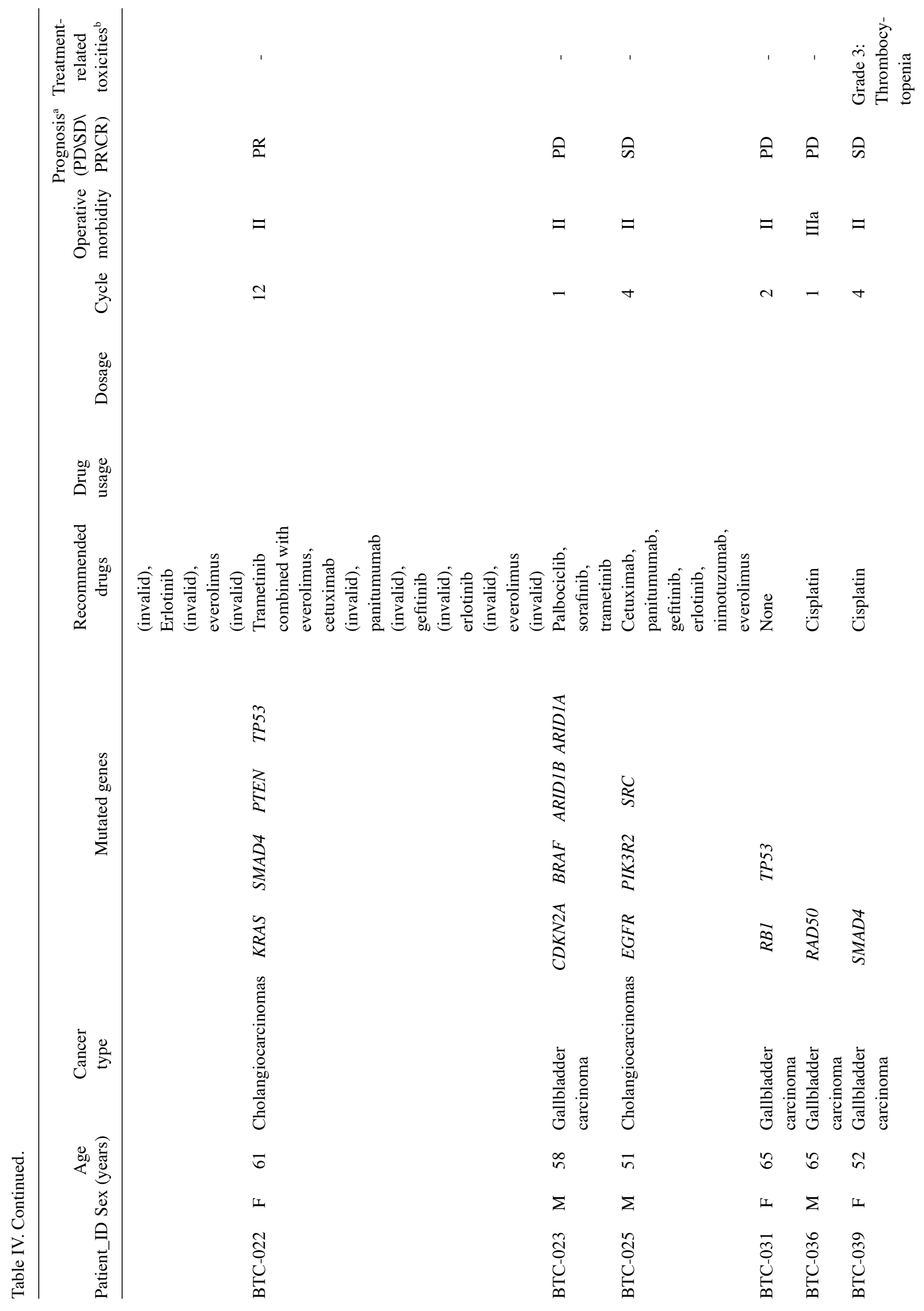




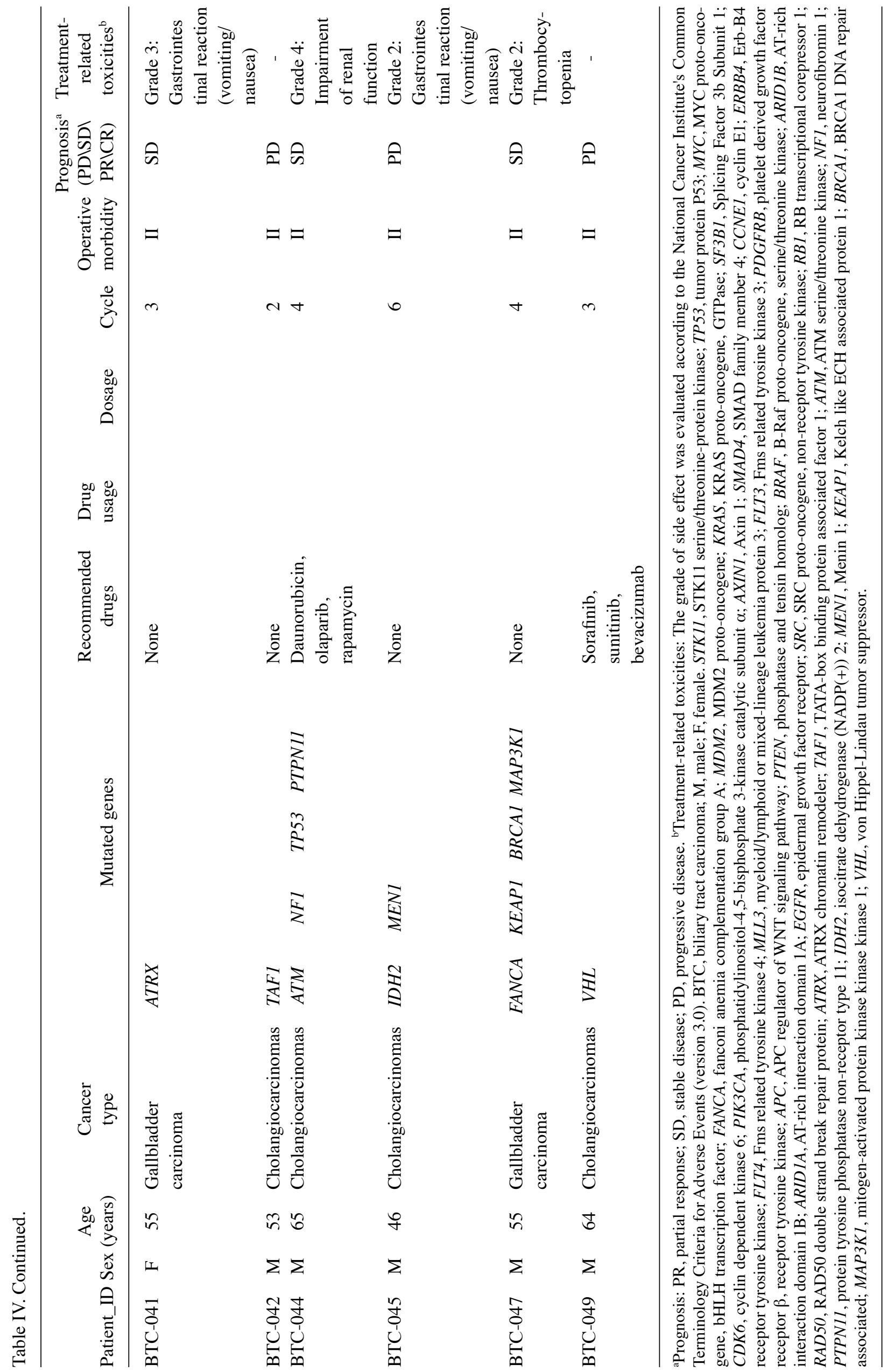




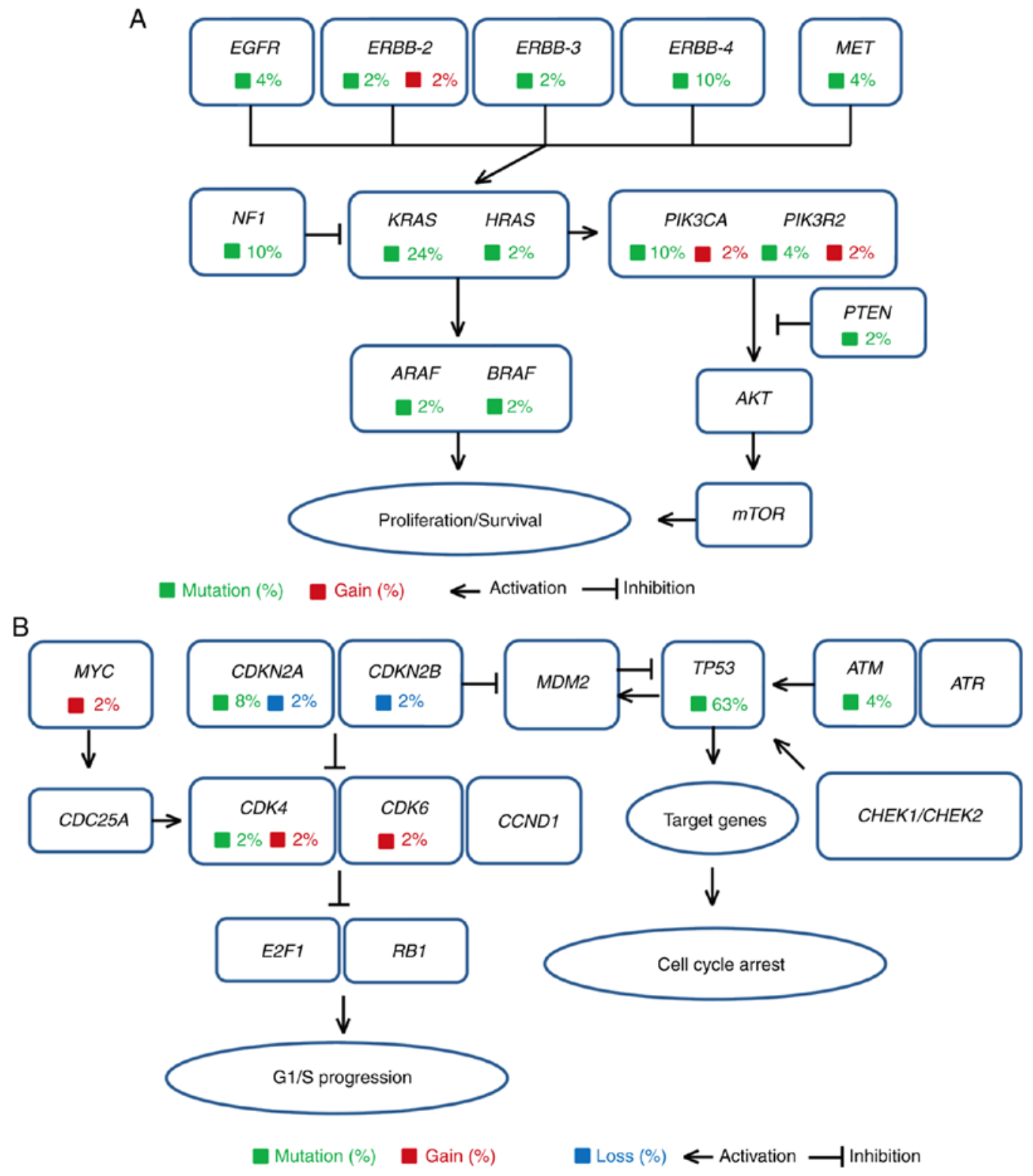

Figure 2. Cellular signaling pathways associated with the mutated genes of the biliary tract cancer (BTC) cases. The mutated genes in 49 patients with BTCs were found to be mainly distributed in the (A) ERBB family signaling pathway and (B) cell cycle signaling pathway. Genes responsible for somatic cell variants and the proportion of mutated genes in 49 patients are indicated in the signal transduction pathway. Different types of variants are marked with different colors, such as mutation (green), gain (red), and loss (blue). EGFR, epidermal growth factor receptor; ERBB2, Erb-B2 receptor tyrosine kinase; MET, MET proto-oncogene, receptor tyrosine kinase; $N F 1$, neurofibromin 1; KRAS, KRAS proto-oncogene, GTPase; HRAS, HRas proto-oncogene, GTPase; PIK3CA, phosphatidylinositol-4,5-bisphosphate 3-kinase catalytic subunit $\alpha ; P I K 3 R 2$, phosphoinositide-3-kinase regulatory subunit 2; ARAF, A-Raf proto-oncogene, serine/threonine kinase; $B R A F$, B-Raf proto-oncogene, serine/threonine kinase; PTEN, phosphatase and tensin homolog; $A K T$, protein kinase B; $m T O R$, mechanistic target of rapamycin kinase; $M Y C$, MYC proto-oncogene, bHLH transcription factor; $C D K N 2 A$, cyclin dependent kinase inhibitor $2 \mathrm{~A} ; C D K N 2 B$, cyclin dependent kinase inhibitor 2B; MDM2, MDM2 proto-oncogene; TP53, tumor protein P53; ATM, ATM serine/threonine kinase; ATR, ATR serine/threonine kinase; $C D C 25 A$, cell division cycle 25A; CDK4, cyclin dependent kinase 4; CDK6, cyclin dependent kinase 6; CCND1, cyclin D1; CHEK1, checkpoint kinase 1; $C H E K 2$, checkpoint kinase 2; E2F1, E2F transcription factor 1; RB1, RB transcriptional corepressor 1.

are consistent with our results. However, we found a higher frequency of $C D K N 2 A$ loss in comparison to Western cohorts (14). High $B R C A$ and $I D H$ mutations were reported in cholangiocarcinoma of Western populations $(3-5,14)$, while no such mutations were found in our study. These aforementioned studies only described the genomic variant landscape and the relationship between prognosis and genomic variants. The use of this genomic profiling information to guide clinical treatment has not been available to use $(14,15)$. Our study focused on advanced BTC patients with non-radical resection, and we assessed the clinical efficacy and safety of personalized targeted therapy guided by targeted deep sequencing in these patients.

In recent years, biomarker-driven clinical trials have been carried out in a wide variety of cancers. Targeted deep sequencing that can achieve high sequencing depth is crucial to accurately identify genomic variants in formalin-fixed paraffin-embedded samples with low tumor cell content and high heterogeneity (32-34), and has also been recognized as a practical method for clinical genetic alteration detection in many types of cancers (35-37). Nevertheless, no studies have been reported on the application of genomic profiling 

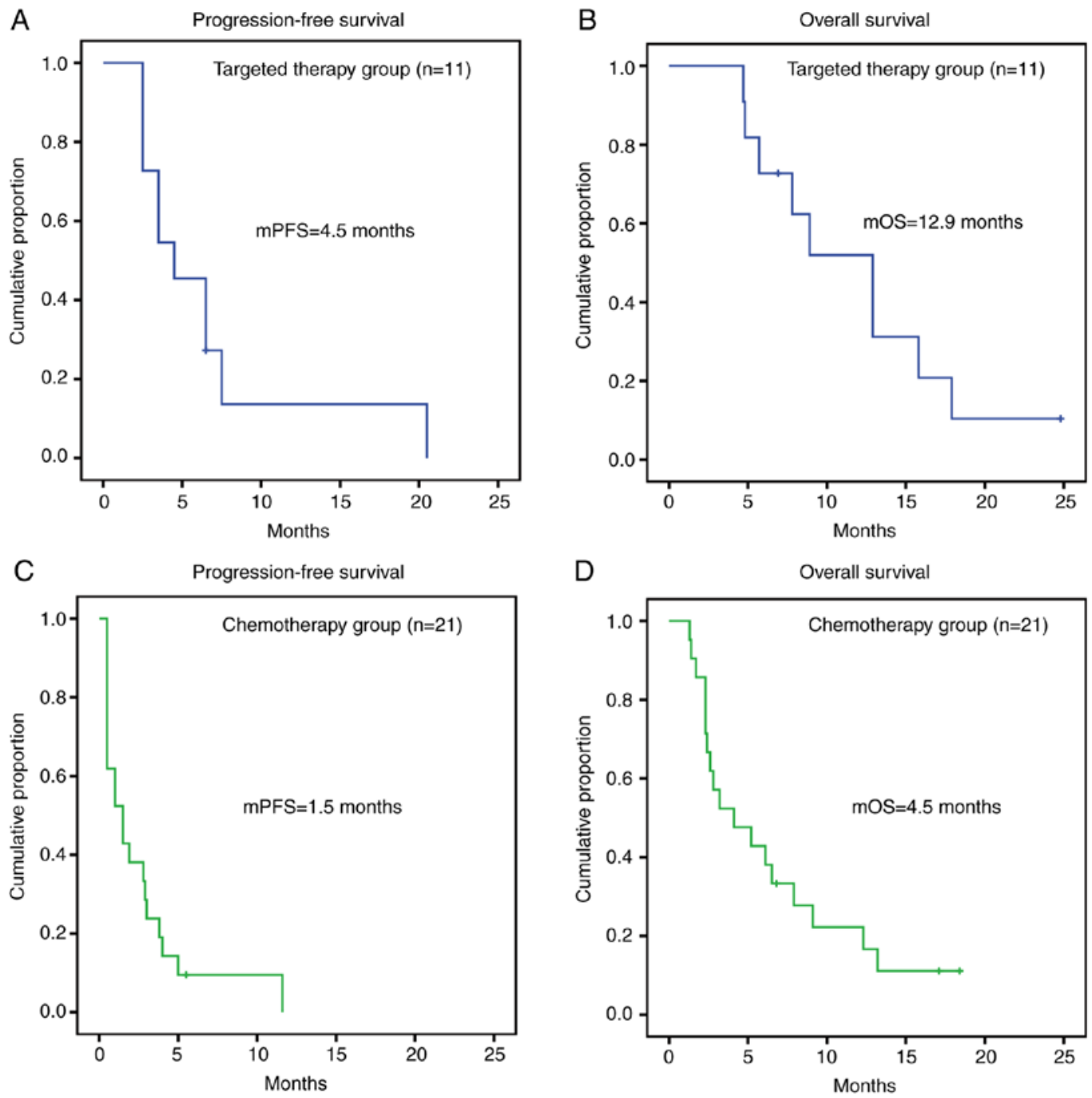

Figure 3. Prognostic analysis of personalized targeted therapy and conventional chemotherapy in patients with biliary tract cancers (BTCs). The genetic variants were detected by targeted deep sequencing in 32 BTC patients with stage IV disease and R2 resection, including 11 patients who received targeted therapy and 21 patients who received conventional chemotherapy. Kaplan-Meier curves of the (A) progression-free survival (PFS) and (B) overall survival (OS) in the targeted therapy group were constructed. Kaplan-Meier curves of the (C) PFS and (D) OS of the chemotherapy group are displayed.

A Lymph-node metastase

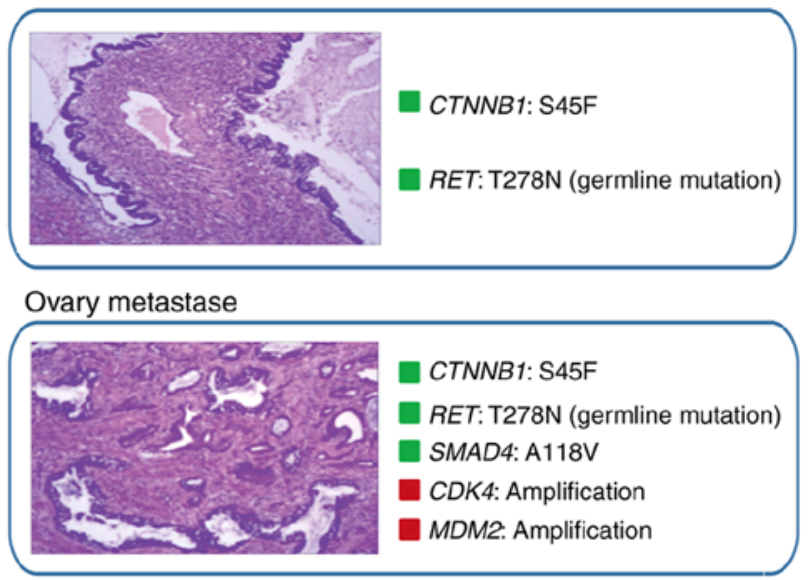

a Substitution/Indel $\square$ Gene amplification
B

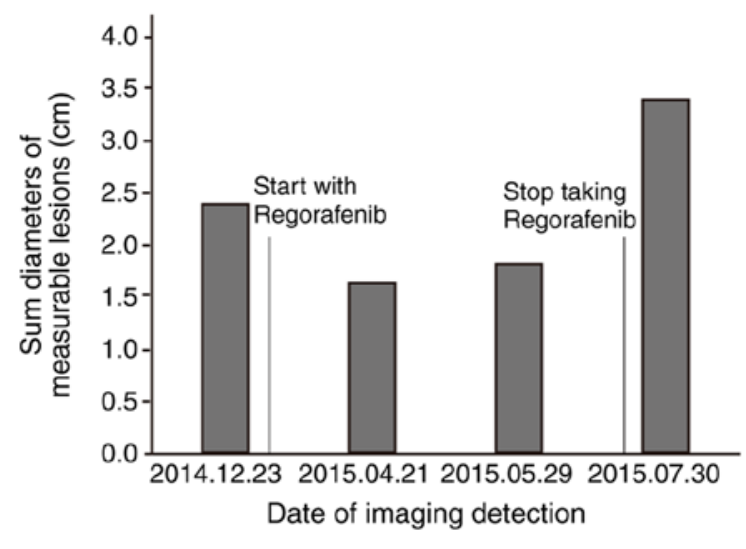

Figure 4. A case of precise therapy for gallbladder carcinoma. (A) Hematoxylin and eosin staining results and genetic variants of metastatic lesions on lymph node and subsequently on the ovary as identified by targeted deep sequencing. Different types of variants are marked with different colors, such as substitution/Indel (green) and gene amplification (red). (B) Sum of diameters of the measurable lesions derived from the four imaging examinations before and after medication. Histogram shows the sum of the diameters of measurable lesions at different dates. CTNNB1, catenin $\beta 1 ; R E T$, Ret proto-oncogene; SMAD4, SMAD family member 4; CDK4, cyclin dependent kinase 4; MDM2, MDM2 proto-oncogene. 
information to guide the precision treatment for a group of advanced BTC patients with non-radical resection. Our study was designed to use targeted deep sequencing for the detection of genetic mutations to guide clinical decision-making in advanced BTC patients with non-radical resection. The personalized targeted therapy group had a median PFS of 4.5 months, a median OS of 12.9 months and a 63.6\% DCR, while the chemotherapy group had a median PFS of 1.5 months, a median OS of 4.1 months, and a $33.3 \%$ DCR. These results may provide preliminary evidence to support the development of a novel treatment strategy of personalized targeted therapy for advanced BTC patients with non-radical resection.

Gemcitabine plus cisplatin (GC) is the standard treatment for advanced BTC for this decade, demonstrating a median OS of gemcitabine regimen of 8.1 months and GC of 11.7 months, respectively (38). The OS of GC reported is longer than that explored in our study. However, there are some differences between their research and ours. Regarding group selection, we focused on the patients with $\mathrm{R} 2$ resection, while they choose patients who did not receive surgery. The two sets of patients are not comparable. The staging system is also different. The 32 patients we used to analyze prognosis were all stage IV patients (with metastatic tumors) in our study, while part of their group was made up of patients with locally advanced cancer but no metastatic tumors. The prognosis of these patients by stage is quite different. Our study more closely reflects real-world clinical practice for advanced BTC with non-radical resection, in which the standardization of drug use and other factors are not as strict as in clinical drug trials.

Treatment-related toxicity is a crucial factor that influences clinical drug use and effects $(39,40)$. In this study, all of the patients in the targeted therapy group experienced Grade 2 or 3 treatment-related toxicities, while five patients in conventional chemotherapy group did. When Grade 2 and 3 toxicity occurred, the drugs could be continuously used by adjusting the drug dosage and drug properties. In both groups, some patients with Grade 2 or 3 toxicities continued taking medicine by reducing the dosage or making other changes. Only one patient in the chemotherapy group with Grade 4 renal impairment stopped taking the drugs. Most patients were on medication regimens for only a short time and the patients in the chemotherapy group did not experience any treatment-related toxicity because of the rapid progression of the disease, which does not mean that these chemotherapeutic drugs had low toxicity. Overall, both targeted therapy and chemotherapy were found to pose some risk of toxicity for BTC patients in real-world clinical practice. The key is finding a way to reduce treatment-related toxicity through drug adjustment or other means so that BTC patients can continue and complete their medication regimens.

In our cohort, 8 of 21 (38.1\%) GBC patients had mutations in the ERBB pathway. It has also been reported that approximately $36.8 \%$ of GBC patients have aberrant ERBB signaling, and multivariate analyses revealed that patients with ERBB pathway mutations had worse outcomes (12). However, there are no clinical studies that have explored if interrogating ERBB signaling can improve the prognoses of such GBC patients. In this study, 3 advanced GBC patients who received therapy specifically targeting alterations in the ERBB pathway achieved marginally longer PFS and OS (Fig. S3), in comparison to the 5 GBC patients who underwent conventional chemotherapy. Despite the small sample size of GBC patients treated with targeted therapy, the preliminary results have shed light on precision therapy for GBC patients with mutations in the ERBB signaling pathway.

Furthermore, we observed that most BTC patients who progressed rapidly such that those in the chemotherapy group experienced only 1-2 cycles of chemotherapy (Table IV). Some BTC patients experienced treatment-related toxicity and had to stop taking chemotherapy drugs. Overall, BTC patients with PR and SD underwent more cycles than those with PD did. Here, BTC-045 was an outlier. BTC-045 had PD and underwent six cycles of chemotherapy. To some extent, chemotherapy cycles are related with disease progression in this real-world clinical study.

The present research has several limitations. Some recommended targeted drugs are difficult to obtain because some of them have been approved by the United States Food and Drug Administration (FDA) but not by the China Food and Drug Administration (CFDA). In consideration of high medical costs, many patients chose the cheaper option, even if another option was favored that may have been more effective. Furthermore, genetic testing did not show which mutations were related to the resistance of chemotherapy in the present study. We do not have access to any other information on BTC patients with targetable altered genes resistant to chemotherapy. In addition, toxicity caused a dose reduction in the targeted drugs, optimization of the medication plan, or finally discontinuation in the medication in this study, which affected the evaluation of the drug effect. Although this real-world clinical study included the greatest number of patients with $\mathrm{R} 2$ resection undergoing personalized precision therapy of any such study, total sample size and the proportion of BTC patients taking the medicine were relatively small. Large umbrella trials of personalized precision therapy are needed to confirm our findings. Despite various limitations, this study reflects real-world clinical practice as it relates to personalized targeted therapy guided by targeted deep sequencing in patients with advanced BTC undergoing non-radical resection in China.

In conclusion, the results of this clinical study suggest that targeted deep sequencing offers a promising method of detecting actionable genetic alterations in BTC cases for precision therapy. This study provides preliminary evidence that personalized targeted therapy based on actionable genetic alterations may benefit patients with advanced BTC undergoing non-radical resection. Large umbrella trials covering personalized precision therapy are needed to confirm the clinical efficacy and safety of this therapeutic strategy for patients with advanced BTC undergoing non-radical resection.

\section{Acknowledgements}

The authors would like to thank Yizhou Ye, Chao Sun, Zishou Zhou, and Xinkai Cao for their assistance with data collection and analysis for this study. In addition, the authors would like to thank all of the patients and their families for their support of this study. 


\section{Funding}

This study is partially supported by the following grants: The Science and Technology Commission of Shanghai Municipality (no. 15411951900), the National Natural Science Foundation of China (no. 81472280), and the Special Fund for the Application and Transformation of Precision Medicine at the Second Military Medical University (no. 2017JZ11).

\section{Availability of data and materials}

The datasets generated and analyzed during the present study are available from the corresponding author on reasonable request.

\section{Authors' contributions}

FF designed the targeted therapy study, contributed to data interpretation and wrote the manuscript. QC designed the targeted therapy study of gallbladder carcinoma patients and wrote the manuscript; DZ designed the targeted deep sequencing study, contributed to data interpretation and composed the manuscript. BL performed the targeted therapy study of gallbladder carcinoma patients and interpreted the data; HQ analyzed the data from targeted deep sequencing and contributed to data interpretation. CX collected tumor tissue from cholangiocarcinoma patients and contributed to data interpretation; $\mathrm{MH}$ analyzed the clinical information and interpreted the data. YY collected the tumor tissue from gallbladder carcinoma patients and collected the clinical information; ZL performed the extraction of genomic DNA from tumor tissue samples and contributed to data interpretation. JYL performed the quality control analyses on targeted deep sequencing and interpreted the data; ZQ performed the targeted therapy study of cholangiocarcinoma patients and interpreted the data. LX discussed the hypothesis, interpreted data, and composed the manuscript; CL performed the construction of sequencing library, analyzed the clinical information of patients, and contributed to data interpretation. FL performed the analyses on the SNV/Indel and copy number variations of tumor tissue samples, contributed to data interpretation, and composed the manuscript; BY led the project, conceived the study, and composed the manuscript; XJ conceived the study and participated in its design and coordination. All authors read and approved the manuscript and agree to be accountable for all aspects of the research in ensuring that the accuracy or integrity of any part of the work are appropriately investigated and resolved.

\section{Ethics approval and consent to participate}

This study protocol was approved by the Institutional Review Board of Shanghai Eastern Hepatobiliary Surgery Hospital, Navy Military Medical University (no. EHBHKY2015-02-010). Written informed consent was obtained from all of the BTC patients.

\section{Patient consent for publication}

Not applicable.

\section{Competing interests}

All of the authors affiliated with 3D Medicines Inc. are current or former employees. The other authors have no potential or actual conflicts of interest to disclose.

\section{References}

1. Marcano-Bonilla L, Mohamed EA, Mounajjed T and Roberts LR: Biliary tract cancers: Epidemiology, molecular pathogenesis and genetic risk associations. Chin Clin Oncol 5: 61, 2016.

2. Streeter OE Jr, Beron PJ and Iyer PN: Precision Medicine: Genomic profiles to individualize therapy. Otolaryngol Clin North Am 50: 765-773, 2017.

3. Xie D, Ren Z, Fan J and Gao Q: Genetic profiling of intrahepatic cholangiocarcinoma and its clinical implication in targeted therapy. Am J Cancer Res 6: 577-586, 2016.

4. Wardell CP, Fujita M, Yamada T, Simbolo M, Fassan M, Karlic R, Polak P, Kim J, Hatanaka Y, Maejima K, et al: Genomic characterization of biliary tract cancers identifies driver genes and predisposing mutations. J Hepatol 68: 959-969, 2018.

5. Andersen JB, Spee B, Blechacz BR, Avital I, Komuta M, Barbour A, Conner EA, Gillen MC, Roskams T, Roberts LR, et al: Genomic and genetic characterization of cholangiocarcinoma identifies therapeutic targets for tyrosine kinase inhibitors. Gastroenterology 142: 1021.e15-1031.e15, 2012.

6. Javle M, Rashid A, Churi C, Kar S, Zuo M, Eterovic AK, Nogueras-Gonzalez GM, Janku F, Shroff RT, Aloia TA, et al: Molecular characterization of gallbladder cancer using somatic mutation profiling. Hum Pathol 45: 701-708, 2014.

7. Papadopoulou K, Murray S, Manousou K, Tikas I, Dervenis C, Sgouros J, Rontogianni D, Lakis S, Bobos M, Poulios C, et al: Genotyping and mRNA profiling reveal actionable molecular targets in biliary tract cancers. Am J Cancer Res 8: 2-15, 2018.

8. Nakamura H, Arai Y, Totoki Y, Shirota T, Elzawahry A, Kato M, Hama N, Hosoda F, Urushidate T, Ohashi S, et al: Genomic spectra of biliary tract cancer. Nat Genet 47: 1003-1010, 2015.

9. Jain A, Kwong LN and Javle M: Genomic profiling of biliary tract cancers and implications for clinical practice. Curr Treat Options Oncol 17: 58, 2016.

10. Zhang H, Berezov A, Wang Q, Zhang G, Drebin J, Murali R and Greene MI: ErbB receptors: From oncogenes to targeted cancer therapies. J Clin Invest 117: 2051-2058, 2007.

11. Tebbutt N,Pedersen MW and Johns TG: Targeting the ERBB family in cancer: Couples therapy. Nat Rev Cancer 13: 663-673, 2013.

12. Li M, Zhang Z, Li X, Ye J, Wu X, Tan Z, Liu C, Shen B, Wang XA, $\mathrm{Wu} \mathrm{W}$, et al: Whole-exome and targeted gene sequencing of gallbladder carcinoma identifies recurrent mutations in the ErbB pathway. Nat Genet 46: 872-876, 2014

13. Kawamoto $\mathrm{T}$, Ishige $\mathrm{K}$, Thomas M, Yamashita-Kashima $\mathrm{Y}$, Shu S, Ishikura N, Ariizumi S, Yamamoto M, Kurosaki K and Shoda J: Overexpression and gene amplification of EGFR, HER2, and HER 3 in biliary tract carcinomas, and the possibility for therapy with the HER2-targeting antibody pertuzumab. J Gastroenterol 50: 467-479, 2015.

14. Javle M, Bekaii-Saab T, Jain A, Wang Y, Kelley RK, Wang K, Kang HC, Catenacci D, Ali S, Krishnan S, et al: Biliary cancer: Utility of next-generation sequencing for clinical management. Cancer 122: 3838-3847, 2016.

15. Sundar R, Custodio A, Petruckevich A, Chénard-Poirier M, Ameratunga M, Collins D, Lim J, Kaye SB, Tunariu N, Banerji U, et al: Clinical outcome of patients with advanced biliary tract cancer in a dedicated phase I unit. Clin Oncol (R Coll Radiol) 30: 185-191, 2018.

16. Sharma A, Dwary AD, Mohanti BK, Deo SV, Pal S, Sreenivas V, Raina V, Shukla NK, Thulkar S, Garg P and Chaudhary SP: Best supportive care compared with chemotherapy for unresectable gall bladder cancer: A randomized controlled study. J Clin Oncol 28: 4581-4586, 2010.

17. Eisenhauer EA, Therasse P, Bogaerts J, Schwartz LH, Sargent D, Ford R, Dancey J, Arbuck S, Gwyther S, Mooney M, et al: New response evaluation criteria in solid tumours: Revised RECIST guideline (version 1.1). Eur J Cancer 45: 228-247, 2009.

18. Briasoulis E, Aravantinos G, Kouvatseas G, Pappas P, Biziota E, Sainis I, Makatsoris T, Varthalitis I, Xanthakis I, Vassias A, et al: Dose selection trial of metronomic oral vinorelbine monotherapy in patients with metastatic cancer: A hellenic cooperative oncology group clinical translational study. BMC Cancer 13: 263, 2013. 
19. Su D, Zhang D, Chen K, Lu J, Wu J, Cao X, Ying L, Jin Q, Ye Y, Xie Z, et al: High performance of targeted next generation sequencing on variance detection in clinical tumor specimens in comparison with current conventional methods. J Exp Clin Cancer Res 36: 121, 2017.

20. Li H and Durbin R: Fast and accurate short read alignment with Burrows-Wheeler transform. Bioinformatics 25: 1754-1760, 2009.

21. Wang Q, Jia P, Li F, Chen H, Ji H, Hucks D, Dahlman KB, Pao W and Zhao Z: Detecting somatic point mutations in cancer genome sequencing data: A comparison of mutation callers. Genome Med 5: 91, 2013

22. Ye K, Schulz MH, Long Q, Apweiler R and Ning Z: Pindel: A pattern growth approach to detect break points of large deletions and medium sized insertions from paired-end short reads. Bioinformatics 25: 2865-2871, 2009.

23. Koboldt DC, Chen K, Wylie T, Larson DE, McLellan MD, Mardis ER, Weinstock GM, Wilson RK and Ding L: VarScan: Variant detection in massively parallel sequencing of individual and pooled samples. Bioinformatics 25: 2283-2285, 2009.

24. Casey BJ, Somerville LH, Gotlib IH, Ayduk O, Franklin NT, Askrend MK, Jonides J, Berman MG, Wilson NL, Teslovich T, et al: Behavioral and neural correlates of delay of gratification 40 years later: Proc Natl Acad Sci USA 108, 14998-5003, 2011.

25. Skidmore ZL, Wagner AH, Lesurf R, Campbell KM, Kunisaki J, Griffith OL and Griffith M: GenVisR: Genomic visualizations in R. Bioinformatics 32: 3012-3014, 2016.

26. Jung W, Jang JY, Kang MJ, Chang YR, Shin YC, Chang J and Kim SW: Effects of surgical methods and tumor location on survival and recurrence patterns after curative resection in patients with T2 gallbladder cancer. Gut Liver 10: 140-146, 2016

27. Shu YJ, Weng H, Bao RF, Wu XS, Ding Q, Cao Y, Wang XA, Zhang F, Xiang SS, Li HF, et al: Clinical and prognostic significance of preoperative plasma hyperfibrinogenemia in gallbladder cancer patients following surgical resection: A retrospective and in vitro study. BMC Cancer 14: 566, 2014.

28. Andersen JB and Thorgeirsson SS: Genetic profiling of intrahepatic cholangiocarcinoma. Curr Opin Gastroenterol 28: 266-272, 2012.

29. Oliveira DV, Zhang S, Chen X, Calvisi DF and Andersen JB: Molecular profiling of intrahepatic cholangiocarcinoma: The search for new therapeutic targets. Expert Rev Gastroenterol Hepatol 11: 349-356, 2017.

30. Chong DQ and Zhu AX: The landscape of targeted therapies for cholangiocarcinoma: Current status and emerging targets. Oncotarget 7: 46750-46767, 2016.
31. Lee H and Ross JS: The potential role of comprehensive genomic profiling to guide targeted therapy for patients with biliary cancer. Therap Adv Gastroenterol 10: 507-520, 2017.

32. Wagle N, Berger MF, Davis MJ, Blumenstiel B, Defelice M, Pochanard P, Ducar M, Van Hummelen P, Macconaill LE, Hahn WC, et al: High-throughput detection of actionable genomic alterations in clinical tumor samples by targeted, massively parallel sequencing. Cancer Discov 2: 82-93, 2012.

33. Klein CJ and Foroud TM: Neurology individualized medicine: When to use next-generation sequencing panels. Mayo Clin Proc 92: 292-305, 2017.

34. LaDuca H, Farwell KD, Vuong H, Lu HM, Mu W, Shahmirzadi L, Tang S, Chen J, Bhide S and Chao EC: Exome sequencing covers $>98 \%$ of mutations identified on targeted next generation sequencing panels. PLoS One 12: e0170843, 2017.

35. Kou T,Kanai M, Yamamoto Y,Kamada M,Nakatsui M,Sakuma T, Mochizuki H, Hiroshima A, Sugiyama A, Nakamura E, et al: Clinical sequencing using a next-generation sequencing-based multiplex gene assay in patients with advanced solid tumors. Cancer Sci 108: 1440-1446, 2017.

36. Ge S, Li B, Li Y, Li Z, Liu Z, Chen Z, Wu J, Gao J and Shen L: Genomic alterations in advanced gastric cancer endoscopic biopsy samples using targeted next-generation sequencing. Am J Cancer Res 7: 1540-1553, 2017.

37. Au CH, Wa A, Ho DN, Chan TL and Ma ES: Clinical evaluation of panel testing by next-generation sequencing (NGS) for gene mutations in myeloid neoplasms. Diagn Pathol 11: 11, 2016.

38. Valle J, Wasan H, Palmer DH, Cunningham D, Anthoney A, Maraveyas A, Madhusudan S, Iveson T, Hughes S, Pereira SP, et al: Cisplatin plus gemcitabine versus gemcitabine for biliary tract cancer. N Engl J Med 362: 1273-1281, 2010.

39. Perović Mihanović M, Haque NS, Rutherford GW, Zekan Š and Begovac J: Toxicity-related antiretroviral drug treatment modifications in individuals starting therapy: A cohort analysis of time patterns, sex, and other risk factors. Med Sci Monit 19: 483-492, 2013.

40. Bar-Ad V, Ohri N and Werner-Wasik M: Esophagitis, treatment-related toxicity in non-small cell lung cancer. Rev Recent Clin Trials 7: 31-35, 2012.

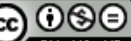

This work is licensed under a Creative Commons Attribution-NonCommercial-NoDerivatives 4.0 International (CC BY-NC-ND 4.0) License. 